\title{
NEW RECIPROCAL SUMS INVOLVING FINITE PRODUCTS OF SECOND ORDER RECURSIONS
}

\author{
EMRAH KILIÇ AND DIDEM ERSANLI
}

Received 15 January, 2019

\begin{abstract}
In this paper, we present new kinds of reciprocal sums of finite products of general second order linear recurrences. In order to evaluate explicitly them by $q$-calculus, first we convert them into their $q$-notation and then use the methods of partial fraction decomposition and creative telescoping.
\end{abstract}

2010 Mathematics Subject Classification: 11B37; 05A30

Keywords: reciprocal sums identities, partial fraction decomposition

\section{INTRODUCTION}

For $n>1$, define the second order linear recurrences $\left\{U_{n}\right\}$ and $\left\{V_{n}\right\}$ with

$$
U_{n}=p U_{n-1}+U_{n-2} \text { and } V_{n}=p V_{n-1}+V_{n-2},
$$

where $U_{0}=0, U_{1}=1$ and $V_{0}=2, V_{1}=p$, respectively.

If $p=1$, then $U_{n}=F_{n}$ ( $n$th Fibonacci number) and $V_{n}=L_{n}$ ( $n$th Lucas number). If $p=2$, then $U_{n}=P_{n}$ ( $n$th Pell number) and $V_{n}=Q_{n}$ ( $n$th Pell-Lucas number).

The Binet forms are

$$
U_{n}=\frac{\alpha^{n}-\beta^{n}}{\alpha-\beta}=\frac{\alpha^{n-1}\left(1-q^{n}\right)}{(1-q)} \text { and } V_{n}=\alpha^{n}+\beta^{n}=\alpha^{n}\left(1+q^{n}\right),
$$

where $\alpha, \beta=\left(p \pm \sqrt{p^{2}+4}\right) / 2, q=\beta / \alpha$ and $\mathbf{i}=\sqrt{-1}$.

Throughout this paper we will use the following notations: the $q$-Pochhammer symbol $(x ; q)_{n}=(1-x)(1-x q) \ldots\left(1-x q^{n-1}\right)$. When $x=q$, we denote $(q ; q)_{n}$ by $(q)_{n}$.

Many authors [1-11] have studied both finite or infinite and alternating or nonalternating reciprocal sums including terms of certain integer sequences. More recently, Frontczak [3] evaluated various reciprocal sums of the Fibonacci numbers. For example, he showed that for $m, n \geq 1$

$$
\sum_{i=1}^{N}(-1)^{m(i+1)} \frac{F_{m i+n \mp 1}}{F_{m(i-1)+n} F_{m i+n} F_{m(i+1)+n}}=\frac{F_{m \mp 1}}{F_{n} F_{m} F_{2 m}}
$$

(c) 2019 Miskolc University Press 


$$
\times\left(\frac{F_{m(N+1)}}{F_{m(N+1)+n}}+\frac{F_{m N}}{F_{m N+n}}-\frac{F_{m}}{F_{m+n}}\right)-(-1)^{m} \frac{F_{m N}}{F_{m}^{2} F_{m+n} F_{m(N+1)+n}} .
$$

Kılıç and Prodinger [5] consider some classes of reciprocal sums of general Fibonacci numbers, which were computed in closed form in an earlier work and then they evaluate the same sums by a different approach: First they convert them into their $q$-forms and then explicitly evaluate $q$-versions of the sums by using partial fraction decomposition method and creative telescoping idea.

In this paper, we shall consider new kinds of reciprocal sums including finite products of the general Fibonacci and Lucas numbers. We shall summarize what we will present in this paper below.

- We consider three kinds of alternating reciprocal sums including finite products of the general Fibonacci and Lucas numbers. All of them have an integer parameter to increase or decrease the indices of the general Fibonacci or Lucas factors in both numerator and denominator in the sums. Two of these sums are of the forms

$$
\sum_{k=0}^{n}(-1)^{k} \frac{U_{k-d}}{U_{k+d} U_{k+d+1} U_{k+d+2}} \text { and } \sum_{k=0}^{n}(-1)^{k} \frac{V_{k+d+1}}{U_{k+d} U_{k+d+1} U_{k+d+2}} \text {. }
$$

The other kind sums has an additional parameter $m$ which determines the number of the general Fibonacci or Lucas factors in the numerator or denominator. It will be of the form

$$
\sum_{k=0}^{n}(-1)^{k} \frac{U_{k+c} U_{k+c+1} \ldots U_{k+c+m-1}}{X_{k+d} X_{k+d+1} \ldots X_{k+d+m+1}},
$$

where $X_{n}$ is either $U_{n}$ or $V_{n}$.

- Before all, we convert all the claimed results into their $q$-forms. After this, we will use partial fraction decomposition (pfd) method and creative telescoping idea to prove the $q$-version of the claimed results.

- By the $q$-versions of three kinds of reciprocal sums, we present general cases of the original three kinds of sums with an additional integer parameter by taking a special choosing of $q$. By the way, we could able to derive nonalternating reciprocal sums where the indices of the general Fibonacci or Lucas numbers are in the arithmetic progressions.

- Finally we shall give some applications of our results to certain alternating and non-alternating reciprocal sums with finite products of the Pell or PellLucas numbers.

\section{THE MAIN RESULTS}

Now we are going to present our main results. We start with our first main result. 
Theorem 1. For $n, m \geq 0$ and $c \in\{-m+1, \ldots,-1,0,1\}$,

$$
\begin{aligned}
& \sum_{k=0}^{n}(-1)^{k} \frac{U_{k+c} U_{k+c+1} \ldots U_{k+c+m-1}}{X_{k+d} X_{k+d+1} \ldots X_{k+d+m+1}} \\
& =(-1)^{c+1} \frac{U_{n+c} U_{n+c+1} \ldots U_{n+c+m}}{U_{m+1} X_{d-c+1}\left[X_{n+d+1} X_{n+d+2} \ldots X_{n+d+m+1}\right]},
\end{aligned}
$$

where $X_{n}$ is either $U_{n}$ or $V_{n}$. Note that when $X_{n}=V_{n}$, we assume that $d$ is any integer. When $X_{n}=U_{n}$, we assume that $d \geq 1$.

Proof. Let $X_{n}=U_{n}$. First we convert the LHS of the claim into its $q$-notation as shown

$$
\sum_{k=0}^{n}(-1)^{k} \frac{\prod_{t=0}^{m-1} U_{k+t+c}}{\prod_{t=0}^{m+1} U_{k+t+d}}=\alpha^{m c-(m+2) d-2 m+1}(1-q)^{2} \sum_{k=0}^{n} \frac{q^{k} \prod_{t=0}^{m-1}\left(1-q^{k+c+t}\right)}{\prod_{t=0}^{m+1}\left(1-q^{k+d+t}\right)} .
$$

Second we convert the RHS of the claim into its $q$-notation as shown

$$
\begin{aligned}
& (-1)^{c+1} \frac{\prod_{t=0}^{m} U_{n+t+c}}{U_{m+1} U_{d-c+1} \prod_{t=1}^{m+1} U_{n+t+d}} \\
& =(-1)^{c+1} \alpha^{(m+2) c-(m+2) d-2 m-1} \frac{(1-q)^{2} \prod_{t=0}^{m}\left(1-q^{n+c+t}\right)}{\left(1-q^{m+1}\right)\left(1-q^{d-c+1}\right) \prod_{t=1}^{m+1}\left(1-q^{n+d+t}\right)} .
\end{aligned}
$$

After some simplifications, the $q$-version of the claimed result is

$$
\sum_{k=0}^{n} \frac{q^{k} \prod_{t=0}^{m-1}\left(1-q^{k+c+t}\right)}{\prod_{t=0}^{m+1}\left(1-q^{k+d+t}\right)}=\frac{q^{1-c} \prod_{t=0}^{m}\left(1-q^{n+c+t}\right)}{\left(1-q^{m+1}\right)\left(1-q^{d-c+1}\right) \prod_{t=1}^{m+1}\left(1-q^{n+d+t}\right)}
$$

or, in terms of the $q$-Pochhammer notation,

$$
\sum_{k=0}^{n} \frac{q^{k}\left(q^{k+c} ; q\right)_{m}}{\left(q^{k+d} ; q\right)_{m+2}}=\frac{q^{1-c}\left(q^{n+c} ; q\right)_{m+1}}{\left(1-q^{m+1}\right)\left(1-q^{d-c+1}\right)\left(q^{n+d+1} ; q\right)_{m+1}}
$$


Define

$$
S_{n}:=\sum_{k=0}^{n} \frac{q^{k}\left(q^{k+c} ; q\right)_{m}}{\left(q^{k+d} ; q\right)_{m+2}}
$$

Denote the summand term of $S_{n}$ by $T_{k}$, that is,

$$
T_{k}=\frac{q^{k}\left(q^{k+c} ; q\right)_{m}}{\left(q^{k+d} ; q\right)_{m+2}}
$$

The partial fraction decomposition of $T_{k}$ reads as

$$
\frac{q^{k}\left(q^{k+c} ; q\right)_{m}}{\left(q^{k+d} ; q\right)_{m+2}}=\sum_{t=1}^{m+2} \frac{q^{-d-t+1}\left(q^{c-d-t+1} ; q\right)_{m}}{\left(1-q^{k+d+t-1}\right) \prod_{\substack{i=1 \\ i \neq t}}^{m+2}\left(1-q^{i-t}\right)}
$$

From [5], we could obtain the following identity comes from creative telescoping idea:

$$
\sum_{k=0}^{n}\left(\frac{1}{1-q^{k+d+m}}-\frac{1}{1-q^{k+d+t}}\right)=\sum_{k=0}^{m-t-1}\left(\frac{1}{1-q^{k+d+n+t+1}}-\frac{1}{1-q^{k+d+t}}\right) .
$$

In that case, by using the equality (2.1), we write

$$
\begin{aligned}
S_{n}= & \left.\sum_{t=1}^{m+1}\left(\frac{1}{1-q^{d+t-1}}-\frac{1}{1-q^{d+n+t}}\right) \sum_{r=1}^{t} \frac{q^{-d-r+1}\left(q^{c-d-r+1} ; q\right)_{m}}{\left(q^{1-r} ; q\right)_{r-1}(q)_{m-r+2}}\right) \\
= & (-1)^{m} \frac{1}{1-q^{m+1}} \sum_{t=1}^{m+1}\left(\frac{1}{1-q^{d+t-1}}-\frac{1}{1-q^{d+n+t}}\right) \\
& \times q^{(m-t+1) c-(m-t+2) d+t(t-1)+\frac{m(m-2 t+1)}{2}} \frac{\left(q^{c-d-2} ; q\right)_{3-t}\left(q^{d-c} ; q\right)_{t-m+1}}{(q)_{t-1}(q)_{m-t+1}} \\
= & (-1)^{m} \frac{1-q^{n+1}}{1-q^{m+1}} \sum_{t=1}^{m+1} \frac{q^{t(-c+d-m+t)}}{\left(1-q^{d+t-1}\right)\left(1-q^{d+n+t)}\right.} \\
& \times q^{c-1-d+\frac{1}{2} m(m+1+2 c-2 d)} \frac{\left(q^{c-d-2} ; q\right)_{3-t}\left(q^{d-c} ; q\right)_{t-m+1}}{(q)_{t-1}(q)_{m-t+1}} \\
= & \frac{1-q^{n+1}}{\left(1-q^{c-d-1}\right)\left(1-q^{m+1}\right)}
\end{aligned}
$$




$$
\begin{aligned}
& \times \sum_{t=1}^{m+1}(-1)^{t+1} \frac{q^{\left(\begin{array}{c}
t+1 \\
2
\end{array}\right)-1}\left(q^{c-d-1} ; q\right)_{2-t}\left(q^{c-d} ; q\right)_{m-t+1}}{\left(1-q^{d+t-1}\right)\left(1-q^{d+n+t}\right)(q)_{t-1}(q)_{m-t+1}} \\
& =\frac{1-q^{n+1}}{\left(1-q^{c-d-1}\right)\left(1-q^{m+1}\right)} \\
& \times \sum_{t=0}^{m}(-1)^{t} \frac{q^{\left(\begin{array}{c}
t+2 \\
2
\end{array}\right)-1}\left(q^{c-d-1} ; q\right)_{1-t}\left(q^{c-d} ; q\right)_{m-t}}{\left(1-q^{d+t}\right)\left(1-q^{d+n+t+1}\right)(q)_{t}(q)_{m-t}} \\
& =\frac{1-q^{n+1}}{\left(1-q^{m+1}\right)\left(1-q^{c-d-1}\right)} \sum_{t=0}^{m}(-1)^{t} \frac{q^{\left(\begin{array}{c}
t \\
2
\end{array}\right)}}{\left(q^{-t}-q^{d}\right)\left(q^{-t}-q^{d+n+1}\right)} \\
& \times \frac{\left(1-q^{c-d-t-1}\right)\left(1-q^{c-d-t}\right) \ldots\left(1-q^{c-d+m-t-2}\right)\left(1-q^{c-d+m-t-1}\right)}{(q)_{t}(q)_{m-t}} \text {. }
\end{aligned}
$$

Now we define

$$
h(z):=\frac{\left(1-z q^{c-d-1}\right) \ldots\left(1-z q^{c-d+m-1}\right)}{\left(z-q^{d}\right)\left(z-q^{d+n+1}\right)(1-z)(1-z q) \ldots\left(1-z q^{m}\right)} .
$$

The partial fraction decomposition of $h(z)$ is

$$
\begin{aligned}
h(z)= & \frac{\left(1-q^{c-1}\right) \ldots\left(1-q^{c+m-1}\right)}{\left(z-q^{d}\right)\left(q^{d}-q^{d+n+1}\right)\left(1-q^{d}\right)\left(1-q^{d+1}\right) \ldots\left(1-q^{d+m}\right)} \\
& +\frac{\left(1-q^{c+n}\right) \ldots\left(1-q^{c+n+m}\right)}{\left(q^{d+n+1}-q^{d}\right)\left(z-q^{d+n+1}\right)\left(1-q^{d+n+1}\right)\left(1-q^{d+n+2}\right) \ldots\left(1-q^{d+n+m+1}\right)} \\
& \left.+\sum_{t=0}^{m}(-1)^{t} q^{\left({ }^{+1}\right.}{ }^{2}\right) \frac{\left(1-q^{-t+c-d-1}\right) \ldots\left(1-q^{-t+c-d+m-1}\right)}{\left(q^{-t}-q^{d}\right)\left(q^{-t}-q^{d+n+1}\right)(q)_{t}(q)_{m-t}\left(1-z q^{t}\right)} .
\end{aligned}
$$

If we multiply $h(z)$ by $z$ and let $z \rightarrow \infty$, then we get

$$
\begin{aligned}
0= & \frac{q^{-d}\left(1-q^{c-1}\right) \ldots\left(1-q^{c+m-1}\right)}{\left(1-q^{n+1}\right)\left(1-q^{d}\right)\left(1-q^{d+1}\right) \ldots\left(1-q^{d+m}\right)} \\
& -\frac{q^{-d}\left(1-q^{c+n}\right) \ldots\left(1-q^{c+n+m}\right)}{\left(1-q^{n+1}\right)\left(1-q^{d+n+1}\right)\left(1-q^{d+n+2}\right) \ldots\left(1-q^{d+n+m+1}\right)} \\
& +\sum_{t=0}^{m}(-1)^{t+1} q^{\left(\begin{array}{l}
t \\
2
\end{array}\right)} \frac{\left(1-q^{-t+c-d-1}\right) \ldots\left(1-q^{-t+c-d+m-1}\right)}{\left(q^{-t}-q^{d}\right)\left(q^{-t}-q^{d+n+1}\right)(q)_{t}(q)_{m-t}} .
\end{aligned}
$$

Since $c \in\{-m+1, \ldots,-1,0,1\}$, we write

$$
\sum_{t=0}^{m}(-1)^{t} q^{\left(\begin{array}{c}
t \\
2
\end{array}\right)} \frac{\left(1-q^{-t+c-d-1}\right) \ldots\left(1-q^{-t+c-d+m-1}\right)}{\left(q^{-t}-q^{d}\right)\left(q^{-t}-q^{d+n+1}\right)(q)_{t}(q)_{m-t}}
$$




$$
=-\frac{q^{-d}\left(1-q^{n+c}\right) \ldots\left(1-q^{c+m+n}\right)}{\left(1-q^{n+1}\right)\left(1-q^{d+n+1}\right)\left(1-q^{d+n+2}\right) \ldots\left(1-q^{d+m+n+1}\right)} .
$$

Taking into the constant factor, we write

$$
\begin{aligned}
& \frac{1-q^{n+1}}{\left(1-q^{m+1}\right)\left(1-q^{c-d-1}\right)} \\
& \quad \times \sum_{t=0}^{m}(-1)^{t} q^{\left(\begin{array}{l}
t \\
2
\end{array}\right)+1} \frac{\left(1-z q^{c-d-1}\right) \ldots\left(1-z q^{c-d+m-1}\right)}{\left(z-q^{d}\right)\left(z-q^{d+n+1}\right)(q)_{t}(q)_{m-t}} \\
& =-\frac{q^{-d}\left(1-q^{n+c}\right) \ldots\left(1-q^{c+m+n}\right)}{\left(1-q^{m+1}\right)\left(1-q^{c-d-1}\right)\left(1-q^{d+n+1}\right) \ldots\left(1-q^{d+m+n+1}\right)} \\
& =-\frac{q^{-d}\left(q^{n+c} ; q\right)_{m+1}}{\left(1-q^{m+1}\right)\left(1-q^{c-d-1}\right)\left(q^{n+d+1} ; q\right)_{m+1}} \\
& =\frac{q^{1-c}\left(q^{n+c} ; q\right)_{m+1}}{\left(1-q^{m+1}\right)\left(1-q^{d-c+1}\right)\left(q^{n+d+1} ; q\right)_{m+1}},
\end{aligned}
$$

which completes the proof.

Also, when $X_{n}=V_{n}$, the proof is similarly obtained.

Now we will present some interesting corollaries of Theorem 1 . When $m=2$, $X_{n}=L_{n}, U_{n}=F_{n}, d=3$ and $c=0$ in Theorem 1, it gives us

$$
\sum_{k=0}^{n}(-1)^{k} \frac{F_{k} F_{k+1}}{L_{k+3} L_{k+4} L_{k+5} L_{k+6}}=-\frac{F_{n} F_{n+1} F_{n+2}}{F_{3} L_{4} L_{n+4} L_{n+5} L_{n+6}} .
$$

When $m=3, X_{n}=U_{n}=P_{n}, d=5$ and $c=1$ in Theorem 1, we get

$$
\sum_{k=0}^{n}(-1)^{k} \frac{P_{k+1} P_{k+2} P_{k+3}}{P_{k+5} P_{k+6} P_{k+7} P_{k+8} P_{k+9}}=\frac{P_{n+1} P_{n+2} P_{n+3} P_{n+4}}{P_{4} P_{5} P_{n+6} P_{n+7} P_{n+8} P_{n+9}} .
$$

When $m=4, X_{n}=Q_{n}, U_{n}=P_{n}, d=4$ and $c=-2$ in Theorem 1, we get

$$
\begin{aligned}
\sum_{k=0}^{n}(-1)^{k} \frac{P_{k-2} P_{k-1} P_{k} P_{k+1}}{Q_{k+4} Q_{k+5} Q_{k+6} Q_{k+7} Q_{k+8} Q_{k+9}} & = \\
& -\frac{P_{n-2} P_{n-1} P_{n} P_{n+1} P_{n+2}}{P_{5} Q_{7} Q_{n+5} Q_{n+6} Q_{n+7} Q_{n+8} Q_{n+9}} .
\end{aligned}
$$

When $m=5, X_{n}=U_{n}=F_{n}, d=5$ and $c=-3$ in Theorem 1, we get

$$
\sum_{k=0}^{n}(-1)^{k} \frac{F_{k-3} F_{k-2} F_{k-1} F_{k} F_{k+1}}{F_{k+5} F_{k+6} F_{k+7} F_{k+8} F_{k+9} F_{k+10} F_{k+11}}
$$




$$
=\frac{F_{n-3} F_{n-2} F_{n-1} F_{n} F_{n+1} F_{n+2}}{F_{6} F_{9} F_{n+6} F_{n+7} F_{n+8} F_{n+9} F_{n+10} F_{n+11}} .
$$

Now we shall give our second result. The first main result stands for the finite product of the general Fibonacci or Lucas numbers. But the next two results are valid for special cases.

Theorem 2. For $d>0$,

$$
\sum_{k=0}^{n}(-1)^{k} \frac{U_{k-d}}{U_{k+d} U_{k+d+1} U_{k+d+2}}=(-1)^{d+1} \frac{U_{2 n+2}}{U_{2} U_{n+d+1} U_{n+d+2}} .
$$

Proof. First we convert the LHS of (2.2) into its $q$-notation as shown

$$
\begin{aligned}
& \sum_{k=0}^{n}(-1)^{k} \frac{U_{k-d}}{U_{k+d} U_{k+d+1} U_{k+d+2}} \\
&=\alpha^{-4 d-1}(1-q)^{2} \sum_{k=0}^{n} \frac{q^{k}\left(1-q^{k-d}\right)}{\left(1-q^{k+d}\right)\left(1-q^{k+d+1}\right)\left(1-q^{k+d+2}\right)} .
\end{aligned}
$$

Second we convert the RHS of (2.2) into its $q$-notation as shown

$$
(-1)^{d+1} \frac{U_{2 n+2}}{U_{2} U_{n+d+1} U_{n+d+2}}=\alpha^{-2 d-1}(1-q)^{2} \frac{(-1)^{d+1}\left(1-q^{2 n+2}\right)}{\left(1-q^{2}\right)\left(1-q^{d+n+1}\right)\left(1-q^{d+n+2}\right)} .
$$

After some simplifications, $q$-version of the claimed result is

$$
\sum_{k=0}^{n} \frac{q^{k}\left(1-q^{k-d}\right)}{\left(1-q^{k+d}\right)\left(1-q^{k+d+1}\right)\left(1-q^{k+d+2}\right)}=-\frac{q^{-d}\left(1-q^{2 n+2}\right)}{\left(1-q^{2}\right)\left(1-q^{d+n+1}\right)\left(1-q^{d+n+2}\right)} .
$$

Define

$$
S_{n}:=\sum_{k=0}^{n} \frac{z\left(1-z q^{-d}\right)}{\left(1-z q^{d}\right)\left(1-z q^{d+1}\right)\left(1-z q^{d+2}\right)} .
$$

Denote the summand term of $S_{n}$ by $T(z)$, that is,

$$
T(z)=\frac{z\left(1-z q^{-d}\right)}{\left(1-z q^{d}\right)\left(1-z q^{d+1}\right)\left(1-z q^{d+2}\right)} .
$$

The partial fraction decomposition of $T(z)$ reads as

$$
\begin{aligned}
& \frac{z\left(1-z q^{-d}\right)}{\left(1-z q^{d}\right)\left(1-z q^{d+1}\right)\left(1-z q^{d+2}\right)} \\
& =\frac{1}{q^{3 d+1}(1-q)^{2}(1+q)}\left(-\frac{q\left(1-q^{2 d}\right)}{1-z q^{d}}+\frac{(1+q)\left(1-q^{2 d+1}\right)}{1-z q^{d+1}}-\frac{1-q^{2 d+2}}{1-z q^{d+2}}\right) \\
& =\frac{1}{q^{3 d+1}(1-q)^{2}(1+q)}\left[q\left(1-q^{2 d}\right)\left(\frac{1}{1-z q^{d+2}}-\frac{1}{1-z q^{d}}\right)\right.
\end{aligned}
$$




$$
\left.-(1+q)\left(1-q^{2 d+1}\right)\left(\frac{1}{1-z q^{d+2}}-\frac{1}{1-z q^{d+1}}\right)\right] .
$$

And so

$$
\begin{aligned}
S_{n}= & \frac{1}{q^{3 d+1}(1-q)^{2}(1+q)}\left[q\left(1-q^{2 d}\right) \sum_{k=0}^{n}\left(\frac{1}{1-z q^{d+2}}-\frac{1}{1-z q^{d}}\right)\right. \\
& \left.-(1+q)\left(1-q^{2 d+1}\right) \sum_{k=0}^{n}\left(\frac{1}{1-z q^{d+2}}-\frac{1}{1-z q^{d+1}}\right)\right] .
\end{aligned}
$$

If we take $m=2, t=0$, and, $m=2, t=1$ in (2.1), respectively, then we rewrite $S_{n}$ given in (2.3) as

$$
\begin{aligned}
& \frac{1}{q^{3 d+1}(1-q)^{2}(1+q)}\left[q\left(1-q^{2 d}\right) \sum_{k=0}^{1}\left(\frac{1}{1-q^{k+d+1+n}}-\frac{1}{1-q^{k+d}}\right)\right. \\
& \left.-(1+q)\left(1-q^{2 d+1}\right)\left(\frac{1}{1-q^{d+n+2}}-\frac{1}{1-q^{d+1}}\right)\right] \\
= & -\frac{\left(1+q^{n+1}\right)\left(1-q^{d+1}\right)\left(1-q^{n+1}\right)}{q^{d}\left(1-q^{d+1}\right)\left(1-q^{d+n+1}\right)\left(1-q^{d+n+2}\right)(1-q)(1+q)} \\
= & -\frac{q^{-d}\left(1-q^{2 n+2}\right)}{\left(1-q^{2}\right)\left(1-q^{d+n+1}\right)\left(1-q^{d+n+2}\right)} .
\end{aligned}
$$

Thus, we have the conclusion.

When $U_{n}=F_{n}$ and $d=3$ in (2.2), we obtain

$$
\sum_{k=0}^{n}(-1)^{k} \frac{F_{k-3}}{F_{k+3} F_{k+4} F_{k+5}}=\frac{F_{2 n+2}}{F_{n+4} F_{n+5}} .
$$

Now we going to give our third result:

Theorem 3. For $d>0$,

$$
\sum_{k=0}^{n}(-1)^{k} \frac{V_{k+d+1}}{U_{k+d} U_{k+d+1} U_{k+d+2}}=\frac{U_{n+1} U_{n+2(d+1)}}{U_{1} U_{d} U_{d+1} U_{n+d+1} U_{n+d+2}} .
$$

Proof. After required converting and simplifications, we find the $q$-version of the claimed result as follows

$$
\begin{aligned}
& \sum_{k=0}^{n} \frac{q^{k}\left(1+q^{k+d+1}\right)}{\left(1-q^{k+d}\right)\left(1-q^{k+d+1}\right)\left(1-q^{k+d+2}\right)} \\
& =\frac{\left(1-q^{n+1}\right)\left(1-q^{n+2(d+1)}\right)}{(1-q)\left(1-q^{d}\right)\left(1-q^{d+1}\right)\left(1-q^{n+d+1}\right)\left(1-q^{n+d+2}\right)} .
\end{aligned}
$$


Define

$$
S_{n}:=\sum_{k=0}^{n} \frac{z\left(1+z q^{d+1}\right)}{\left(1-z q^{d}\right)\left(1-z q^{d+1}\right)\left(1-z q^{d+2}\right)} .
$$

Denote the summand term of $S_{n}$ by $T(z)$, that is,

$$
T(z)=\frac{z\left(1+z q^{d+1}\right)}{\left(1-z q^{d}\right)\left(1-z q^{d+1}\right)\left(1-z q^{d+2}\right)} .
$$

The partial fraction decomposition of $T(z)$ reads as

$$
\begin{aligned}
& \frac{z\left(1+z q^{d+1}\right)}{\left(1-z q^{d}\right)\left(1-z q^{d+1}\right)\left(1-z q^{d+2}\right)} \\
& =\frac{1}{q^{d}(1+q)(1-q)^{2}}\left(\frac{1+q}{1-z q^{d}}-\frac{2(1+q)}{1-z q^{d+1}}+\frac{1+q}{1-z q^{d+2}}\right) \\
& =\frac{-1}{q^{d}(1+q)(1-q)^{2}}\left[(1+q)\left(\frac{1}{1-z q^{d+2}}-\frac{1}{1-z q^{d}}\right)\right. \\
& \left.-2(1+q)\left(\frac{1}{1-z q^{d+2}}-\frac{1}{1-z q^{d+1}}\right)\right] .
\end{aligned}
$$

And so

$$
\begin{aligned}
S_{n}= & \frac{-1}{q^{d}(1+q)(1-q)^{2}}\left[(1+q) \sum_{k=0}^{n}\left(\frac{1}{1-z q^{d+2}}-\frac{1}{1-z q^{d}}\right)\right. \\
& \left.-2(1+q) \sum_{k=0}^{n}\left(\frac{1}{1-z q^{d+2}}-\frac{1}{1-z q^{d+1}}\right)\right] .
\end{aligned}
$$

If we take $m=2, t=0$, and, $m=2, t=1$ in (2.1), respectively, then we rewrite the last equality as

$$
\begin{aligned}
S_{n}= & \frac{-1}{q^{d}(1+q)(1-q)^{2}}\left[(1+q) \sum_{k=0}^{1}\left(\frac{1}{1-q^{k+d+1+n}}-\frac{1}{1-q^{k+d}}\right)\right. \\
& \left.-2(1+q)\left(\frac{1}{1-q^{d+n+2}}-\frac{1}{1-q^{d+1}}\right)\right] \\
& =\frac{\left(1-q^{n+1}\right)\left(1-q^{n+2 d+2}\right)}{(1-q)\left(1-q^{d}\right)\left(1-q^{d+1}\right)\left(1-q^{n+d+1}\right)\left(1-q^{n+d+2}\right)} .
\end{aligned}
$$

Thus, we have the conclusion.

When $U_{n}=F_{n}, V_{n}=L_{n}$ and $d=5$ in (2.4), we obtain

$$
\sum_{k=0}^{n}(-1)^{k} \frac{L_{k+6}}{F_{k+5} F_{k+6} F_{k+7}}=\frac{F_{n+1} F_{n+12}}{F_{5} F_{6} F_{n+6} F_{n+7}} \text {. }
$$




\section{General CASES}

In the previous section, we found the $q$-versions of the claimed results in Theorems 1-3 while proving them. Now we shall present more general cases of Theorems $1-3$ by taking $q=\beta^{s} / \alpha^{s}$ for any integer $s$ in the $q$-forms of them without proof, respectively.

Theorem 4. For $n, m \geq 0$ and $c \in\{-m+1, \ldots,-1,0,1\}$,

$$
\sum_{k=0}^{n}(-1)^{s k} \frac{\prod_{t=0}^{m-1} U_{s(k+c+t)}}{\prod_{t=0}^{m+1} X_{s(k+d+t)}}=(-1)^{s(c+1)} \frac{\prod_{t=0}^{m} U_{s(n+t+c)}}{U_{s(m+1)} X_{s(d-c+1)} \prod_{t=1}^{m+1} X_{s(n+t+d)}},
$$

where $X_{n}$ is either $U_{n}$ or $V_{n}$. Note that when $X_{n}=V_{n}$, we assume that $d$ is any integer. When $X_{n}=U_{n}$, we assume that $d \geq 1$.

In (3.1), if we take $m=3, s=2, U_{n}=P_{n}, X_{n}=Q_{n}, d=4$ and $c=1$, then we obtain

$\sum_{k=0}^{n} \frac{P_{2 k+2} P_{2 k+4} P_{2 k+6}}{Q_{2 k+8} Q_{2 k+10} Q_{2 k+12} Q_{2 k+14} Q_{2 k+16}}=\frac{P_{2 n+2} P_{2 n+4} P_{2 n+6} P_{2 n+8}}{P_{8} Q_{8} Q_{2 n+10} Q_{2 n+12} Q_{2 n+14} Q_{2 n+16}}$.

When $m=4, s=-1, U_{n}=X_{n}=F_{n}, d=6$ and $c=0$ in Theorem 4, then, by $F_{-n}=(-1)^{n+1} F_{n}$, we obtain

$$
\begin{aligned}
& \sum_{k=0}^{n}(-1)^{k} \frac{F_{-k} F_{-k-1} F_{-k-2} F_{-k-3}}{F_{-k-6} F_{-k-7} F_{-k-8} F_{-k-9} F_{-k-10} F_{-k-11}} \\
& =\sum_{k=0}^{n}(-1)^{k} \frac{F_{k} F_{k+1} F_{k+2} F_{k+3}}{F_{k+6} F_{k+7} F_{k+8} F_{k+9} F_{k+10} F_{k+11}} \\
& =-\frac{F_{n} F_{n+1} F_{n+2} F_{n+3} F_{n+4}}{F_{5} F_{7} F_{n+7} F_{n+8} F_{n+9} F_{n+10} F_{n+11}} .
\end{aligned}
$$

When $m=5, s=3, U_{n}=F_{n}, X_{n}=L_{n}, d=2$ and $c=-3$ in Theorem 4, then we obtain

$$
\begin{aligned}
& \sum_{k=0}^{n}(-1)^{k} \frac{F_{3 k-9} F_{3 k-6} F_{3 k-3} F_{3 k} F_{3 k+3}}{L_{3 k+6} L_{3 k+9} L_{3 k+12} L_{3 k+15} L_{3 k+18} L_{3 k+21} L_{3 k+24}} \\
& =\frac{F_{3 n-9} F_{3 n-6} F_{3 n-3} F_{3 n} F_{3 n+3} F_{3 n+6}}{F_{18} L_{18} L_{3 n+9} L_{3 n+12} L_{3 n+15} L_{3 n+18} L_{3 n+21} L_{3 n+24}} .
\end{aligned}
$$


Theorem 5. For $d>0$,

$$
\sum_{k=0}^{n}(-1)^{s k} \frac{U_{s(k-d)}}{U_{s(k+d)} U_{s(k+d+1)} U_{s(k+d+2)}}=(-1)^{s d+1} \frac{U_{s(2 n+2)}}{U_{2 s} U_{s(n+d+1)} U_{s(n+d+2)}} \text {. }
$$

If we take $U_{n}=F_{n}, d=2$ and $s=5$ in (3.2), then we obtain

$$
\sum_{k=0}^{n}(-1)^{k} \frac{F_{5 k-10}}{F_{5 k+10} F_{5 k+15} F_{5 k+20}}=-\frac{F_{10 n+10}}{F_{10} F_{5 n+15} F_{5 n+20}} .
$$

Theorem 6. For $d>0$,

$\sum_{k=0}^{n}(-1)^{s k} \frac{V_{s(k+d+1)}}{U_{s(k+d)} U_{s(k+d+1)} U_{s(k+d+2)}}=\frac{U_{s(n+1)} U_{s(n+2 d+2)}}{U_{s} U_{s d} U_{s(d+1)} U_{s(n+d+1)} U_{s(n+d+2)}}$.

If we take $U_{n}=P_{n}, V_{n}=Q_{n}, d=4$ and $s=-3$ in (3.3), then, by $P_{-n}=$ $(-1)^{n+1} P_{n}$ and $Q_{-n}=(-1)^{n} Q_{n}$, we obtain

$$
\begin{aligned}
\sum_{k=0}^{n}(-1)^{k} \frac{Q_{-3 k-15}}{P_{-3 k-12} P_{-3 k-15} P_{-3 k-18}} & =\sum_{k=0}^{n}(-1)^{k} \frac{Q_{3 k+15}}{P_{3 k+12} P_{3 k+15} P_{3 k+18}} \\
& =\frac{P_{3 n+3} P_{3 n+30}}{P_{3} P_{12} P_{15} P_{3 n+15} P_{3 n+18}} .
\end{aligned}
$$

\section{Conclusions}

In the last section, we derived more general results, where the sign functions and the indices of Fibonacci or Lucas factors are depend on the integer parameter $s$. By the way, one can see that these generalized reciprocal sums are alternating for odd integer $s$, and, non-alternating for even integer $s$ while the original sums in Theorems 1-3 are always alternating sums.

\section{REFERENCES}

[1] R. André-Jeannin, "Summation of reciprocals in certain second-order recurring sequences." Fibonacci Quart., vol. 35, no. 1, pp. 68-73, 1997.

[2] G. Choi and Y. Choo, "On the reciprocal sums of square of generalized bi-periodic Fibonacci numbers." Miskolc Math. Notes, vol. 19, no. 1, pp. 201-209, 2018, doi: 10.18514/MMN.2018.2390.

[3] R. Frontczak, "Closed-form evaluations of Fibonacci-Lucas reciprocal sums with three factors." Notes on Number Theory and Discrete Mathematics, vol. 23, no. 2, pp. 104-116, 2017.

[4] E. Kılıç and T. Arıkan, "More on the infinite sum of reciprocal Fibonacci, Pell and higher order recurrences." Appl. Math. Comput., vol. 219, no. 14, pp. 7783-7788, 2013, doi: 10.1016/j.amc.2013.02.003.

[5] E. Kılıç and H. Prodinger, "Closed form evaluation of Melham's reciprocal sums." Miskolc Math. Notes, vol. 18, no. 1, pp. 251-264, 2017, doi: 10.18514/mmn.2017.1284. 
[6] R. Stanley, "Algorithmic simplification of reciprocal sums," in Applications of Fibonacci Numbers. Springer, 1999, pp. 277-292.

[7] G. Xi, "Summation of reciprocals related to $l$-th power of generalized Fibonacci sequences." Ars Combin., vol. 83, pp. 179-191, 2007.

[8] G. Xi, "Reciprocal sums of quadruple product of generalized binary sequences with indices." Util. Math., vol. 97, pp. 321-328, 2015.

[9] G. Xi, "Reciprocal sums of quintuple product of generalized binary sequences with indices." $J$. Comb. Math. Comb. Comput., vol. 101, pp. 37-46, 2017.

[10] L. Xin and L. Xiaoxue, "A reciprocal sum related to the Riemann $\zeta$-function." J. Math. Inequal., vol. 11, no. 1, pp. 209-215, 2017, doi: 10.7153/jmi-11-20.

[11] H. Zhang and Z. Wu, "On the reciprocal sums of the generalized Fibonacci sequences." Adv. Difference Equ., vol. 2013, p. 377, 2013, doi: 10.1186/1687-1847-2013-377.

Authors' addresses

\section{Emrah Kılıç}

TOBB University of Economics and Technology, Mathematics Department, 06560, Ankara, Turkey

E-mail address: ekilicdetu.edu.tr

\section{Didem Ersanlı}

TOBB University of Economics and Technology, Mathematics Department, 06560, Ankara, Turkey

E-mail address: didemersanli@gmail.com 